\title{
Failure to maintain equivalence of groups in cognitive research: Evidence from dual-task methodology
}

\author{
F. RICHARD FERRARO \\ University of North Dakota, Grand Forks, North Dakota \\ and \\ GEORGE KELLAS and GREG B. SIMPSON \\ University of Kansas, Lawrence, Kansas
}

\begin{abstract}
Poulton (1982) has criticized within-subject manipulations of the standard conditions for dualtask methodology. Two experiments compared between- versus within-subject designs employing lexical decisions. The results confirmed Poulton's claim of asymmetry of transfer within repeated measure designs. Conclusions regarding attention allocation, therefore, are equivocal in comparison with independent groups designs.
\end{abstract}

The dual-task procedure is often used to assess the resources required of a cognitive task. In this research, a subject performs (1) the primary task in isolation, (2) the secondary task in isolation, and (3) the primary and secondary tasks together (e.g., Fisk, Derrick, \& Schneider, 1986; Kantowitz, 1974; Kerr, 1973). Secondary task performance from the combined primary- secondary-task condition is thought to reflect the amount of attentional resources demanded by the particular primary task under investigation.

Typically, this research is performed using a withinsubject design, and subjects received all three task conditions (see, e.g., Becker, 1976; Herdman \& Dobbs, 1989; Paap \& Ogden, 1981). However, Poulton (1982) has discussed potential problems associated with withinsubject manipulations in cognitive research. Briefly, Poulton (1982) warns that transfer effects may not always be equivalent across the various conditions. In the kind of dual-task paradigm discussed above, for instance, performance in the combined-task condition may vary according to its position in the experimental sequence (e.g., whether it precedes or follows the single-task conditions). Because of this potential problem, Kellas, Ferraro, and Simpson (1988), in a study investigating the attention demands of word recognition, tested independent groups of subjects in the three task conditions. The primary task in this study was a lexical (word/nonword) decision one in which ambiguous (multiple-meaning) words, unambiguous words, and pseudowords were compared. The secondary task was tone detection. Kellas, Ferraro, and Simpson (1988) found that ambiguous words led both to

These data were presented at the 1990 Annual Meeting of the Psychonomic Society in New Orleans. Address correspondence to F. R. Ferraro, Department of Psychology, University of North Dakota, Grand Forks, ND 58202 (e-mail: ferraro@ndsuvm1.bitnet). faster lexical decision times and to faster tone detection than did unambiguous words or pseudowords. It was also observed that attentional demands decreased across the time course of word recognition.

The following experiment tested the claims made by Poulton (1982) and directly assessed the impact that differential transfer effects may have in research employing within-subject designs such as that reported in Kellas, Simpson, and Ferraro (1988). A portion of the present research replicated the effects found in Kellas, Simpson, and Ferraro, but manipulated task condition within subjects and varied the order in which the three tasks were performed. Each subject performed the lexical decision and tone-detection tasks in isolation and in combination. The order of the three tasks was counterbalanced primarily to investigate the performance in the combined task condition as a function of its position in the sequence of tasks. If Poulton's claims are correct, performance observed when dual-task conditions are manipulated within subjects should differ from performance observed when these same conditions are manipulated between subjects.

\section{METHOD}

\begin{abstract}
Subjects
A total of 36 University of Kansas undergraduates participated for course credit. None had taken part in Kellas, Ferraro, and Simpson's (1988) study. Twelve subjects were each randomly assigned to one of the three experimental conditions as they arrived at the laboratory. All were native English speakers with normal or corrected-to-normal vision and hearing.
\end{abstract}

\section{Materials and Equipment}

Materials and equipment were the same as in Kellas, Ferraro, and Simpson's (1988) study.

\section{Block Design}

The three task conditions (primary alone, secondary alone, and primary and secondary) were systematically counterbalanced across blocks and 
trials. The counterbalancing scheme was such that each task was allowed to occur first, second, and third across the course of the experiment. The design permitted an overall evaluation of the within-subject manipulation of task as well as an evaluation of task as a between-subject variable across the first occurrence of each task. The task orderings were as follows: Task Order 1, primary task-combined task-secondary task; Task Order 2, combined task-secondary task-primary task; Task Order 3, secondary task-primary task-combined task.

Twelve subjects each were randomly assigned to one of the three possible orderings. It should be noted that with the three condition orderings listed above, it is possible to attempt a replication of the original Kellas, Ferraro, and Simpson (1988) between-subject manipulation of the dual-task conditions (i.e., the first task from each of the three orders)

\section{Procedure}

In each task ordering, the subjects received 80 experimental trials in each of the three conditions, for a total of 240 experimental trials. Each separate condition was preceded by 4 buffer trials and 10 practice trials. Short (1-2 $\mathrm{min})$ rest breaks preceded each separate condition, and no feedback regarding response time or error rate was provided. In the primary-task condition, the subjects were told that they would see a string of letters appear in the tachistoscope and should quickly and accurately decide whether or not the string of letters was a real word. Keys were provided for this lexical decision. In the secondary-task condition, the subjects were instructed to press a key whenever they heard a tone through the headphones that they were wearing. In the combined-task condition, the subjects were told that they would see a string of letters on the tachistoscope and hear a tone presented over the headphones. Across trials, the tone could appear either 90,180 , or $270 \mathrm{msec}$ after the onset of the letter string, which represents a manipulation in stimulus onset asynchrony (SOA) between onset of the primary task and onset of the secondary task. The subjects were told to quickly and accurately respond to the tone and then make their lexical decision. The entire experiment lasted between 90 and $100 \mathrm{~min}$.

\section{RESULTS}

Prior to reporting results based on potential strategy transfer effects, it is important to demonstrate two critical outcomes. First, and in accordance with Kerr (1973), it is important to demonstrate primary-task invariance. Performance in the primary task in isolation should not differ from performance in the primary task in conjunction with the secondary task (i.e., the combined-task condition). Second, performance in the combined-task condition (when performed first in the three-condition task order) should replicate that reported by Kellas, Ferraro, and Simpson (1988). When the combined task is performed first in the three-condition task order, there is no previous condition to bias performance. Within the present experiment, it is possible to test these two critical outcomes.

\section{Between-Subject Analysis}

To properly interpret dual-task performance, it is necessary to demonstrate primary-task invariance. In the present experiment, the task invariance analysis was achieved by comparing primary-task performance when it was performed first in the three-task ordering (i.e., Order 1) with primary task performance in the combinedtask condition when it was also performed first in the three-task ordering (i.e., Order 2). This analysis was performed on mean lexical decision latencies and was a 2 (condition: alone and combined) $\times 3$ (stimuli: ambiguous, unambiguous, and pseudoword) mixed-factor anal- ysis of variance (ANOVA), with condition manipulated between subjects. Results revealed no main effect of condition $[F(1,22)=1.22, p=.28]$, a main effect of stimuli $[F(2,44)=58.87, p<.01]$, and no interaction of condition $\times$ stimuli $[F(2,44)=2.41, p=.10]$. The main effect of stimuli replicates that reported by Kellas, Ferraro, and Simpson (1988) by showing that in lexical decisions, ambiguous words are responded to faster than are unambiguous words, which are responded to faster than are pseudowords. Given that condition was neither a significant main effect nor part of a significant interaction, this analysis supports the existence of primary-task invariance, because primary-task performance did not change as a function of combining it with the secondary task (see, e.g., Kerr, 1973). It should also be noted that error rates increased in ascending order from ambiguous words to unambigous words to pseudowords.

Probe-alone performance. The probe-alone performance data was analyzed with a 3 (order) $\times 3$ (SOAs: 90,180 , and $270 \mathrm{msec}$ ) mixed-factor ANOVA, with order the between-subject factor and time the within-subject factor. Results revealed no main effect of order $[F(2,33)=$ $1.95, p=.158]$ and no main effect of time $(F<1.00)$. The interaction of these two factors was also not reliable $(F<2.00)$.

Combined task performance. This analysis looked exclusively at the combined-task performance when it was performed first in the three-task ordering (Order 2). Tone latency from the combined condition was the dependent variable, and this analysis was a 3 (stimuli: ambiguous, unambiguous, and pseudoword) $\times 3$ (SOAs: 90,180 , and $270 \mathrm{msec}$ ) within-subject ANOVA. The results replicated those of Kellas, Ferraro, and Simpson (1988) and revealed a main effect for stimuli $[F(2,22)=26.63, p<.01]$ and a main effect for SOA $[F(2,22)=114.08, p<.01]$. There was also an interaction of these two factors $[F(4,44)$ $=3.91, p<.0084]$. These results indicate that ambiguous words require fewer attentional resources for processing than do unambiguous words and pseudowords, and that processes associated with word recognition require less attention as their processing time course increases.

\section{Within-Subject Analysis}

In this analysis, separate analyses were performed on both the primary-task performance and the secondary-task performance from the combined-task condition. The primary-task analysis was an order $(1,2$, and 3$) \times$ condition (alone and combined) $\times$ stimuli (ambiguous, unambiguous, and pseudoword) mixed-factor ANOVA, with order and condition the between-subject factors. Results revealed main effects for condition $[F(1,66)=8.29, p<$ $.01]$ and stimuli $[F(2,132)=122.62, p<.01]$ but not for order $(F<2.00, p>.20)$. There were interactions involving stimuli $\times$ order $[F(4,132)=8.53, p<.01]$, stimuli $\times$ condition $[F(2,132)=4.84, p<.01]$ but not order $\times$ condition $(F<1.00)$. The most noteworthy outcome of this analysis was the reliable order $\times$ condition $\times$ stimuli interaction $[F(4,132)=3.82, p<.01]$. Fig- 

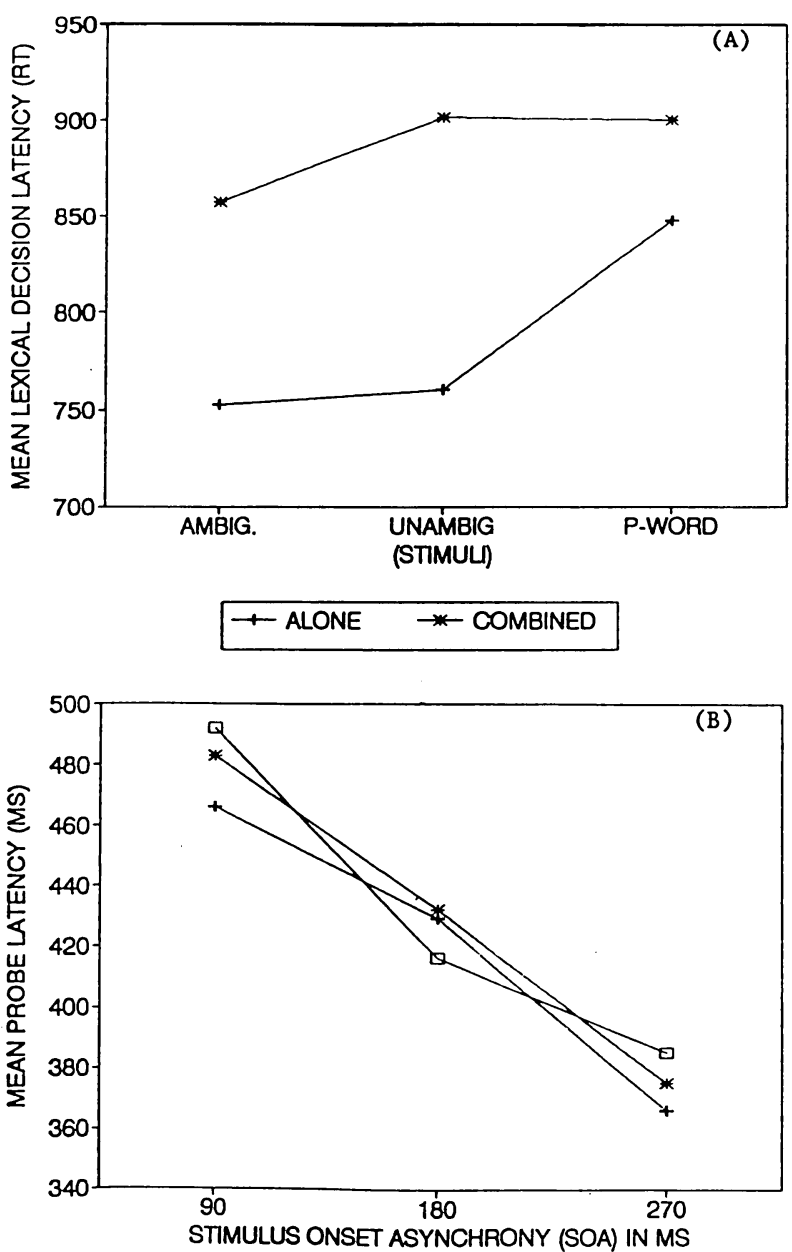

$\rightarrow$ AMBIGUOUS $*$ - UNAMBIGUOUS $\because-$ PSEUDOWORD

Figure 1. (A) Mean lexical decision latencies (in milliseconds) as a function of stimuli type from the third position of condition orders 2 and 3. (B) Mean probe latencies (in milliseconds) as a function of stimuli type and stimulus onset asynchrony from the third position of condition order 3 .

ure 1 (panel A) depicts this interaction at the third order position. As can be seen in Figure 1, not only is primarytask invariance lost, so too is the effect of stimulus type, especially when the primary task was performed in isolation. Although one could argue that this pattern is the result of subject fatigue, this is unlikely because Kellas, Ferraro, and Simpson (1988) did not find such a pattern although they did have more than one block of trials (i.e., block in the Kellas, Ferraro, and Simpson experiment is similar to order in the current experiment).

Probe-alone performance. An analysis was also performed on the probe-alone data, with time as the only factor. This analysis revealed a nonsignificant effect of time $[F(2,22)=2.01, p=.1585]$.

Combined-task performance. We now turn to the analysis performed on probe latencies from the combined-task condition. This analysis was an order $(1,2$, and 3$) \times$ stim- uli (ambiguous, unambiguous, and pseudoword) $\times$ SOAs $(90,180$, and $270 \mathrm{msec}$ ) mixed-factor ANOVA, with order the between-subject factor. This analysis revealed main effects for order $[F(2,33)=3.57, p<.04]$, stimuli $[F(2,66)$ $=30.17, p<.01]$, and time $[F(2,66)=193.60, p<$ $.01]$. There were two-way interactions involving stimuli $\times$ order $[F(4,66)=7.06, p<.01]$. The remaining twoway interactions were not significant (both $F \mathrm{~s}<2.00$ ). Again, the most noteworthy effect was the reliable order $\times$ stimuli $\times$ SOA interaction $[F(8,132)=2.58, p<.02]$. Figure 1 (panel B) depicts this interaction as a function of the third order position. As is clear from Figure 1, the stimuli effect that is typically found (e.g., Kellas, Ferraro, \& Simpson, 1988) is now virtually abolished, with probe latency responses differing little between ambiguous, unambiguous, and pseudoword stimuli. Again, this effect is probably not caused by subject fatigue.

\section{DISCUSSION}

Manipulation of dual-task conditions in a within-subject design adversely affected performance, compared with when the same conditions were manipulated between subjects. This manipulation affected both primary-task and secondary-task performance from the combined-task condition. In accordance with Poulton's (1982) study, the subjects receiving the dual-task conditions in a within-subject design may have altered their processing strategies across the various conditions or continued to employ a strategy that they assumed to be most effective regardless of the condition they were exposed to. Therefore, the asymmetric transfer of processing strategy appears to be a potential problem associated with experiments in which treatment conditions are manipulated within subjects, at least within the context of the present experiment. Further work is needed to pinpoint the locus of these potential strategy influences during the processing sequence, and the current results are a step in this direction.

\section{REFERENCES}

BECKER, C. A. (1976). Allocation of attention during visual word recognition. Journal of Experimental Psychology: Human Perception \& Performance, 2, 556-566.

Fisk, A. D., Derrick, W. L., \& Schneider, W. (1986). A methodological assessment and evaluation of dual-task paradigms. Current Psychological Research \& Reviews, 5, 315-327.

Herdman, C. M., \& DobBs, A. R. (1989). Attentional demands of visual word recognition. Journal of Experimental Psychology: Human Perception \& Performance, 15, 124-132.

Kantowitz, B. H. (1974). Double stimulation. In B. H. Kantowitz (Ed.), Human information processing: Tutorials in performance and cognition (pp. 83-131). Hillsdale, NJ: Erlbaum.

Kellas, G., Ferraro, F. R., \& Simpson, G. B. (1988). Lexical ambiguity and the timecourse of attentional allocation in word recognition. Journal of Experimental Psychology: Human Perception \& Performance, 14, 601-609.

Kellas, G., Simpson, G. B., \& Ferraro, F. R. (1988). Aging and performance: A mental workload analysis. In P. Whitney \& R. Ochsman (Eds.), Psychology and productivity (pp. 35-49). New York: Plenum

KerR, B. (1973). Processing demands during mental operations. Memory \& Cognition, 1, 401-412.

PAAP, K. R., \& OGDEN, W. C. (1981). Letter encoding is an obligatory but capacity-demanding activity. Journal of Experimental Psychology: Human Perception \& Performance, 7, 518-527.

Poulton, E. C. (1982). Influential companions: Effects of one strategy on another in within-subjects designs in cognitive psychology. Psychological Bulletin, 91, 673-690.

(Manuscript received March 26, 1993.) 